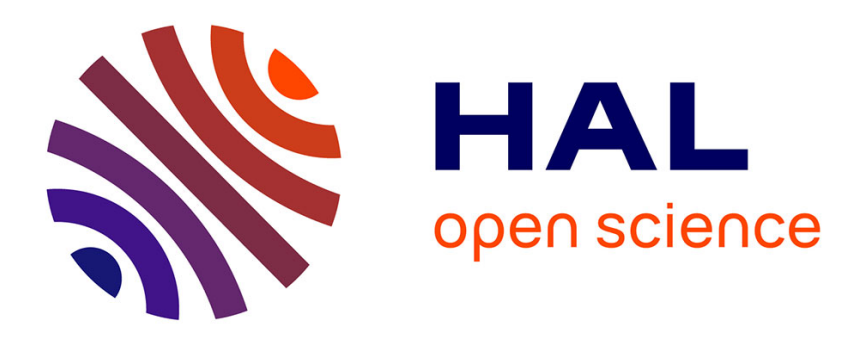

\title{
Extrinsic radius pinching in space forms of nonnegative sectional curvature
}

Julien Roth

\section{To cite this version:}

Julien Roth. Extrinsic radius pinching in space forms of nonnegative sectional curvature. Mathematische Zeitschrift, 2008, 258 (1), pp.227-240. hal-00095786

\section{HAL Id: hal-00095786 \\ https://hal.science/hal-00095786}

Submitted on 18 Sep 2006

HAL is a multi-disciplinary open access archive for the deposit and dissemination of scientific research documents, whether they are published or not. The documents may come from teaching and research institutions in France or abroad, or from public or private research centers.
L'archive ouverte pluridisciplinaire HAL, est destinée au dépôt et à la diffusion de documents scientifiques de niveau recherche, publiés ou non, émanant des établissements d'enseignement et de recherche français ou étrangers, des laboratoires publics ou privés. 


\title{
EXTRINSIC RADIUS PINCHING IN SPACE FORMS OF NONNEGATIVE SECTIONAL CURAVTURE
}

\author{
JULIEN ROTH
}

\author{
Institut Élie Cartan, UMR 7502 \\ Nancy-Université, CNRS, INRIA \\ B.P. 239, 54506 Vandœuvre lès Nancy Cedex, France \\ roth@iecn.u-nancy.fr
}

\begin{abstract}
We first give new estimates for the extrinsic radius of compact hypersurfaces of the Euclidean space $\mathbb{R}^{n+1}$ and the open hemisphere in terms of high order mean curvatures. Then we prove pinching results corresponding to theses estimates. We show that under a suitable pinching condition, $M$ is diffeomorphic and almost isometric to an $n$-dimensional sphere.
\end{abstract}

Key words: Extrinsic radius, pinching, hypersurfaces, $r$-th mean curvature

Mathematics Subject Classification: 53A07, 53C20, 53C21

\section{INTRODUCTION}

Let $\left(M^{n}, g\right)$ be a compact, connected and oriented $n$-dimensional Riemannian manifold without boundary isometrically immersed by $\phi$ into the $(n+1)$-dimensional simply connected space-form $\left(\mathbb{M}^{n+1}(\delta), g_{\text {can }}\right)$ of sectional curvature $\delta$ with $n \geqslant 2$. The extrinsic radius of $(M, g)$ is the real number defined as follows

$$
R=R(M)=\inf \left\{r>0 \mid \exists x \in \mathbb{M}^{n+1}(\delta) \text { s.t. } \phi(M) \subset B(x, r)\right\},
$$

where $B(x, r)$ (resp. $\bar{B}(x, r)$ and $S(x, r))$ is the open ball (resp. the closed ball and the sphere) of center $x$ and radius $r$ in $\mathbb{M}^{n+1}(\delta)$. An immediate consequence of the above definition is that there exists $p_{0} \in$ $\mathbb{M}^{n+1}(\delta)$ such that

$$
\phi(M) \subset \bar{B}\left(p_{0}, R\right) \quad \text { and } \quad \phi(M) \cap S\left(p_{0}, R\right) \neq \emptyset .
$$

Moreover, the extrinsic radius is bounded from below in terms of the mean curvature. More precisely, we have the following estimate obtained by comparing the shape operators for hypersurfaces that have a contact point and where one is outside the other (see [2, 1, 14] for 
details)

where $t_{\delta}(s)= \begin{cases}\frac{1}{\sqrt{\delta}} \tan (\sqrt{\delta} s) & \text { if } \delta>0 \\ s & \text { if } \delta=0 \\ \frac{1}{\sqrt{-\delta}} \tanh (\sqrt{-\delta} s) & \text { if } \delta<0\end{cases}$

and $H$ is the mean curvature of the immersion. Note that if $\delta>0$, the image $\phi(M)$ is assumed to be contained in a ball of radius less than $\frac{\pi}{2 \sqrt{\delta}}$ which is equivalent to the fact that $M$ lies in the open hemisphere $\mathbb{S}_{+}^{n+1}(\delta)$.

Since the equality case is characterized by the geodesic spheres of radius $R$, the question of the associated pinching problem was asked, i.e., what happens in the case of almost equality.

Many pinching results are known for intrinsic geometric invariants defined on Riemannian manifold with positive Ricci curvature, as the intrinsic diameter, the volume, the radius or the first eigenvalue of the Laplacian ( «17, 19, 9, 8, 10, 23, 3]).

Nethertheless, few results are known about pinching problems in the extrinsic case. In [7], B. Colbois and J.F. Grosjean give a first result about the first eigenvalue $\lambda_{1}(M)$ of the Laplacian. More precisely, they proved that there exists a constant $C$ depending on $n$ and the $L^{\infty}$-norm of the second fundamental form such that if

$$
\frac{n}{V(M)^{1 / p}}\|H\|_{2 p}^{2}-C<\lambda_{1}(M)
$$

then $M$ is diffeomorphic to an $n$-dimensional sphere.

We gave a second extrinsic pinching result in 21]. We proved the pinching result associated with Inequality (11), that is, there exists a constant $C$ depending on the $L^{\infty}$-norm of the second fundamental form so that if

$$
t_{\delta}(R)<\frac{1}{\|H\|_{\infty}}+C,
$$

then $M$ is diffeomorphic and almost isometric to a geodesic sphere of radius $R$.

In this paper, we extend the results in 21] to high order mean curvatures $H_{k}$, which are the natural generalization of the mean curvature $H$. They are defined to be the $k$-th elementary symmetric polynomial in the principal curvature of $M$ (see Section 2). For instance, for hypersurfaces of $\mathbb{R}^{n+1}$, up to a multiplicative constant, $H_{1}$ is the mean curvature, $\mathrm{H}_{2}$ is the scalar curvature and $H_{n}$ is the Gauss-Kronecker curvature. These curvatures give, in general, better inequalities than those involving the mean curvature $H$. Indeed, R.C. Reilly gave in [20] a sharper upper bound for the first eigenvalue of the Laplacian for hypersurfaces of $\mathbb{R}^{n+1}$. The analogue of this upper bound was proved 
by J.F. Grosjean for hypersurfaces of $\mathbb{S}^{n+1}$ and $\mathbb{H}^{n+1}$ (see $\llbracket 12 \|$ ).

Regarding the extrinsic radius, T. Vlachos improved Inequality (1) in terms of $H_{k}$, i.e.,

$$
t_{\delta}(R)^{k} \geqslant \frac{1}{\left\|H_{k}\right\|_{\infty}}
$$

with equality only for the geodesic spheres of radius $R$ (see [22]). This is an improvement of Inequality (11) since we have the following sequence of inequalities

$$
H_{k}^{1 / k} \leqslant \cdots \leqslant H_{2}^{1 / 2} \leqslant H .
$$

A first question is to know if Inequality (2) can be improved, replacing the $L^{\infty}$-norm by an $L^{p}$-norm, as for (11) (see [21] for details).

We prove, in Section 3, that such $L^{p}$-lower bounds are true for $\delta \geqslant 0$ and any $k \in\{1, \cdots, n\}$ if $H_{k}$ is a positive function. Moreover, the equality is characterized by geodesic hyperspheres. Then, another natural question, is to know if there exists a pinching result for these inequalities. That is, is there a constant $C$ such that if the pinching condition

$$
t_{\delta}(R)^{k}<\frac{1}{\left\|H_{k}\right\|_{\infty}}+C
$$

holds, then $M$ is closed to a geodesic hypersphere?

In what follows, we denote by $\mathcal{M}(n, \delta, R, k)$ the family of all compact, connected and oriented $n$-dimensional Riemannian manifolds without boundary isometrically immersed into $\mathbb{R}^{n+1}$ if $\delta=0$ or into the open hemisphere of $\mathbb{S}^{n+1}(\delta)$ if $\delta>0$, of extrinsic radius $R$, volume equal to 1 and positive $H_{k}$. We prove the following

Theorem 1. Let $\left(M^{n}, g\right) \in \mathcal{M}(n, \delta, R, k), \delta \geqslant 0$ and $p_{0}$ be the center of the ball of radius $R$ containing $M$. Then for any $\varepsilon>0$, there exists a positive constant $C_{\varepsilon}$ depending only on $n, \delta$, the $L^{\infty}$-norm of the mean curvature and the $L^{2 p}$-norm of the $k$-th mean curvature $H_{k}$ such that if

$$
t_{\delta}(R)^{k}<\frac{1}{\left\|H_{k}\right\|_{2 p}}+C_{\varepsilon}
$$

then

i) $\phi(M) \subset B\left(p_{0}, R\right) \backslash B\left(p_{0}, R-\varepsilon\right)$.

ii) $\forall x \in S\left(p_{0}, R\right), \quad B(x, \varepsilon) \cap \phi(M) \neq \emptyset$.

\section{Remarks}

1) We will see in the proof that $C_{\varepsilon} \longrightarrow 0$ when $\|H\|_{\infty} \longrightarrow \infty$ or $\varepsilon \longrightarrow 0$.

2) An immediate consequence of $i$ ) and $i i$ ) of Theorem 1 is that the Haussdorff-distance between $M$ and $S\left(p_{0}, R\right)$ satisfies

$$
d_{H}\left(M, S\left(p_{0}, R\right)\right) \leqslant \varepsilon
$$


If the pinching condition is strong enough, with a control on the $L^{\infty}$-norm of the second fundamental form $B$ instead of the $L^{\infty}$-norm of the mean curvature, we obtain that $M$ is diffeomorphic and almost isometric to a geodesic sphere in the following sense:

Theorem 2. Let $\left(M^{n}, g\right) \in \mathcal{M}(n, \delta, R, k), \delta \geqslant 0$ and $p_{0}$ be the center of the ball of radius $R$ containing $M$. Then there exists a constant $C$ depending only on $n, \delta$, the $L^{\infty}$-norm of the second fundamental form and the $L^{2 p}$-norm of the $k$-th mean curvature $H_{k}$ such that if

$$
t_{\delta}(R)^{k}<\frac{1}{\left\|H_{k}\right\|_{2 p}}+C
$$

then $M$ is diffeomorphic to $S\left(p_{0}, R\right)$.

More precisely, there exists a diffeomorphism $F$ from $M$ into the geodesic hypersphere $S\left(p_{0}, R\right)$ which is a quasi-isometry. That is, for all $\theta \in] 0,1\left[\right.$, there exist a constant $C_{\theta}$ depending on $n, \delta,\|B\|_{\infty},\left\|H_{k}\right\|_{2 p}$ and $\theta$ such that the pinching condition $\left(P_{C}\right)$ implies

$$
\left.|| d F_{x}(u)\right|^{2}-1 \mid \leqslant \theta
$$

for all unit vector $u \in T_{x} M$.

\section{Remarks.}

1) It is obvious that the pinching condition

$$
t_{\delta}(R)^{k}<\frac{1}{\left\|H_{k}\right\|_{\infty}}+C
$$

implies $\left(P_{C}\right)$. So we deduce immediately from Theorems 11 and 2 the same results with the second pinching condition $\left(\widetilde{P_{C}}\right)$.

2) In general, the constants $C_{\varepsilon}$ and $C_{\theta}$ of Theorems 1 and 2 depend on $\left\|H_{k}\right\|_{2 p}$. In fact, the constant $C_{\varepsilon}$ of Theorem 1 does not depend on $\left\|H_{k}\right\|_{2 p}$ when

(a) $\delta>0$

(b) $\delta=0$ and $k \geqslant 4$,

(c) $\delta=0$ and $p \geqslant \frac{n}{2 k}$,

and the constant $C_{\theta}$ of Theorem 2 does not depend on $\left\|H_{k}\right\|_{2 p}$ when $\delta=0$ and $p \geqslant \frac{n}{2 k}$.

3) Our approach does not work in the case $\delta<0$. As we will see, it is due to the fact that the function $c_{\delta}$ is increasing if $\delta<0$.

4) By homothety, we can deduce the same results for manifolds with arbitrary volume. Indeed, $\left(M, g^{\prime}\right) \in \mathcal{M}\left(n, \delta^{\prime}, R^{\prime}\right)$, with $g^{\prime}=V(M)^{-2 / n} g$, $\delta^{\prime}=V(M)^{2 / n} \delta$ and $R^{\prime}=V(M)^{-1 / n} R$.

These results are of special interest for $k=2$. Indeed, in that case, up to a constant, $\mathrm{H}_{2}$ is the scalar curvature. So we obtain a relation between the extrinsic radius and the scalar curvature, which is an intrinsic geometric invariant. In particular, we have the following corollary 
Corollary 1. Let $\left(M^{n}, g\right) \in \mathcal{M}(n, 0, R)$ and $p_{0}$ be the center of the ball of radius $R$ containing $M$. We assume that $\left(M^{n}, g\right)$ has positive scalar curvature. Then for any $p \geqslant \frac{n}{4}$, there exists a constant $C$ depending only on $n$ and $\|B\|_{\infty}$ such that if

$$
R^{2}<\frac{n(n-1)}{\| \text { Scal } \|_{2 p}}+C
$$

then $M$ is diffeomorphic and almost isometric to $S\left(p_{0}, R\right)$ in the sense of Theorem 2

Acknowledgement. The author would like to express his gratefulness to his advisors Jean-François Grosjean and Oussama Hijazi for their encouragement.

\section{PreliminRaries}

First, let's introduce the following functions:

$$
s_{\delta}(t)= \begin{cases}\frac{1}{\sqrt{\delta}} \sin (\sqrt{\delta} t) & \text { if } \delta>0 \\ t & \text { if } \delta=0\end{cases}
$$

and

$$
c_{\delta}(t)= \begin{cases}\cos (\sqrt{\delta} t) & \text { if } \delta>0 \\ 1 & \text { if } \delta=0\end{cases}
$$

Throughout this paper, we consider a manifold $\left(M^{n}, g\right) \in \mathcal{M}(n, \delta, R, k)$, $\delta \geqslant 0$. For simplicity, we assume that $\delta=0$ or 1 . By homothety, we can deduce the results for any $\delta \geqslant 0$. Let $\nu$ be the outward unit vector field. The second fundamental form $B$ of the immersion is defined by

$$
B(X, Y)=\left\langle\bar{\nabla}_{X} \nu, Y\right\rangle,
$$

where $\langle\cdot, \cdot\rangle$ and $\bar{\nabla}$ are respectively the Riemannian metric and the Riemannian connection of $\mathbb{M}^{n+1}(\delta)$. The mean curvature of the immersion is

$$
H=\frac{1}{n} \operatorname{tr}(B) .
$$

Now let's recall the definition of the high order mean curvature $H_{k}$. Let $\left\{e_{1}, \cdots, e_{n}\right\}$ be an orthonormal frame of $T_{x} M$. For all $k \in$ $\{1, \cdots, n\}$, the $k$-th mean curvature of the immersion is

$$
H_{k}=\left(\begin{array}{c}
n \\
k
\end{array}\right)_{\substack{1 \leqslant i_{1}, \cdots, i_{k} \leqslant n \\
1 \leqslant j_{1}, \cdots, j_{k} \leqslant n}}^{-1} \epsilon\left(\begin{array}{c}
i_{1} \cdots i_{k} \\
j_{1} \cdots j_{k}
\end{array}\right) B_{i_{1} j_{1}} \cdots B_{i_{k} j_{k}},
$$

where the $B_{i j}$ are the coefficients of the real second fundamental form. The symbols $\epsilon\left(\begin{array}{c}i_{1} \cdots i_{k} \\ j_{1} \cdots j_{k}\end{array}\right)$ are the usual premutation symbols which are zero if the sets $\left\{i_{1}, \cdots, i_{k}\right\}$ and $\left\{j_{1}, \cdots, j_{k}\right\}$ are different or if there 
exist distinct $p$ and $q$ with $i_{p}=i_{q}$. For all other cases, $\epsilon\left(\begin{array}{c}i_{1} \cdots i_{k} \\ j_{1} \cdots j_{k}\end{array}\right)$ is the signature of the permutation $\left(\begin{array}{c}i_{1} \cdots i_{k} \\ j_{1} \cdots j_{k}\end{array}\right)$. By convention, we set $H_{0}=1$ et $H_{n+1}=0$.

For $k \in\{1, \cdots, n\}$, the symmetric $(1,1)$-tensor associated to $H_{k}$ is

$$
T_{k}=\frac{1}{k !} \sum_{\substack{1 \leqslant i, i_{1}, \cdots, i_{k} \leqslant n \\
1 \leqslant j, j_{1}, \cdots, j_{k} \leqslant n}} \epsilon\left(\begin{array}{c}
i_{1} \cdots i_{k} \\
j_{1} \cdots j_{k}
\end{array}\right) B_{i_{1} j_{1}} \cdots B_{i_{k} j_{k}} e_{i}^{*} \otimes e_{j}^{*} .
$$

This tensor is divergence free, symmetric $(1,1)$-tensor. For any symmetric (1,1)-tensor, we define the following function

$$
H_{T}(x)=\sum_{i=1}^{n} B_{x}\left(T e_{i}, e_{i}\right),
$$

where $\left\{e_{1}, \ldots, e_{n}\right\}$ is an orthonormal frame of $T_{x} M$. Then, we have the following relations

Lemma 2.1. For $k \in\{1, \cdots, n\}$, we have:

(1) $\operatorname{tr}\left(T_{k}\right)=m(k) H_{k}$,

(2) $H_{T_{k}}=m(k) H_{k+1}$,

where $m(k)=(n-k)\left(\begin{array}{c}n \\ k\end{array}\right)$ and $H_{T_{k}}$ is given by (3).

Let $p_{0} \in \mathbb{M}^{n+1}(\delta)$. We denote by $r(x)=d\left(p_{0}, x\right)$ the geodesic distance from $p_{0}$ to $x$ on $\left(\mathbb{M}^{n+1}(\delta), g_{\text {can }}\right)$. We denote by $\nabla$ (resp. $\bar{\nabla})$ the gradient associated with $(M, g)\left(\operatorname{resp} .\left(\mathbb{M}^{n+1}(\delta), g_{\text {can }}\right)\right)$. Let $Z:=s_{\delta}(r) \bar{\nabla} r$ be the postion vector field and $Z^{T}=s_{\delta}(r) \nabla r$ its tangential projection on the tangent bundle of $\phi(M)$. We have the following lemma (see [15] or 13] for a proof):

Lemma 2.2. Let $T$ be a positive definite $(1,1)$-tensor with $\operatorname{div}(T)=0$. Then we have

$$
\operatorname{div}\left(T Z^{T}\right) \geqslant c_{\delta}(r) \operatorname{tr}(T)-\left\langle Z, H_{T}\right\rangle .
$$

If $\delta=0$ and $T$ is the identity, then equality holds.

Remark. Note that, by integration, in the case $\delta=0$ and $T=\mathrm{Id}$, this lemma is nothing else but the Hsiung-Minkowski formula (see [16]).

Finally, we recall the following lemma (see [7] or [21])

Lemma 2.3. Let $\left(M^{n}, g\right)$ be a compact, connected, oriented $n$-dimensional Riemannian manifold without boundary isometrically immersed by $\phi$ 
into $\mathbb{R}^{n+1}$ or an open hemisphere of $\mathbb{S}^{n+1}(\delta)$. Let $\xi$ be a nonnegative continuous function on $M$ such that $\xi^{k}$ is smooth for $k \geqslant 2$. Let $0 \leqslant l<m \leqslant 2$ such that

$$
\frac{1}{2} \xi^{2 k-2} \Delta \xi^{2} \leqslant \operatorname{div} \omega+\left(\alpha_{1}+k \alpha_{2}\right) \xi^{2 k-l}+\left(\beta_{1}+k \beta_{2}\right) \xi^{2 k-m},
$$

where $\omega$ is a 1-form and $\alpha_{1}, \alpha_{2}, \beta_{1}$ and $\beta_{2}$ some nonnegative constants. Then for all $\eta>0$, there exists a constant $L$ depending only on $\alpha_{1}, \alpha_{2}$, $\beta_{1}, \beta_{2},\|H\|_{\infty}$ and $\eta$ such that if $\|\xi\|_{\infty}>\eta$, then

$$
\|\xi\|_{\infty} \leqslant L\|\xi\|_{2} \text {. }
$$

Moreover, $L$ is bounded when $\eta \longrightarrow \infty$ and if $\beta_{1}>0, L \longrightarrow \infty$ when $\|H\|_{\infty} \longrightarrow \infty$ or $\eta \longrightarrow 0$.

\section{NeW ESTIMATES FOR THE EXTRINSIC RADIUS}

In this section, we give new lower bounds for the extrinsic radius of hypersurfaces of the Euclidean space and the open hemisphere $\mathbb{S}_{+}^{n+1}(\delta)$. Let $\left(M^{n}, g\right)$ be a compact, connected and oriented $n$-dimensional Riemannian manifold without boundary isometrically immersed by $\phi$ in $\mathbb{R}^{n+1}$ or $\mathbb{S}_{+}^{n+1}(\delta)$. An immediate consequence of Lemma 2.2 is the following

Proposition 3.1. Let $k \in\{1, \cdots, n\}$, if $H_{k}$ is positive, then for all $j \in\{1, \cdots, k\}$, the function $H_{j}$ is positive and

$$
\int_{M} H_{j-1} c_{\delta}(r) d v_{g} \leqslant \int_{M} H_{j} s_{\delta}(r) d v_{g}
$$

Proof: By Lemma 2.2, we have

$$
\begin{aligned}
\int_{M} c_{\delta}(r) \operatorname{tr}(T) d v_{g} & \leqslant \int_{M}\left|\left\langle Z, H_{T}\right\rangle\right| d v_{g} \\
& \leqslant \int_{M}\left|H_{T}\right| s_{\delta}(r) d v_{g}
\end{aligned}
$$

If $T=T_{j-1}$ is the $(1,1)$-tensor associated with $H_{j-1}$, we have

$$
\int_{M} c_{\delta}(r) H_{j-1} d v_{g} \leqslant \int_{M}\left|H_{j}\right| s_{\delta}(r) d v_{g}
$$

Moreover, Barbosa and Colares proved in [5] that if $H_{k}$ is positive, then for all $j \in\{1, \cdots, k\}, H_{j}$ is positive. So for all $j \in\{1, \cdots, k\}$, we have

$$
\int_{M} H_{j-1} c_{\delta}(r) d v_{g} \leqslant \int_{M} H_{j} s_{\delta}(r) d v_{g}
$$

From this proposition, we deduce the following estimates 
Theorem 3. Let $\left(M^{n}, g\right) \in \mathcal{M}(n, \delta, R, k)$ (we do not assume that $V(M)=1)$. Then for all $p \geqslant 1$, we have

$$
t_{\delta}(R)^{k} \geqslant \frac{V(M)^{1 / p}}{\left\|H_{k}\right\|_{p}} .
$$

Equality occurs if and only if $M$ is a geodesic hypersphere.

\section{Remarks.}

i) These lower bounds improve Inequalities (11) and (2).

ii) In the case $k=2$, this inequality 4 translates to

$$
\begin{gathered}
R^{2} \geqslant \frac{n(n-1)}{\| \text { Scal } \|_{1}}, \text { if } \delta=0 \\
\tan ^{2}(R) \geqslant \frac{n(n-1)}{\| \text { Scal }-n(n-1) \|_{1}}, \text { if } \delta=1 .
\end{gathered}
$$

Inequaliy (5) was proved by S. Deshmukh ([11). In the spherical case, our inequality improves the following one due to L.J Alias (匹1)

$$
\sin ^{2}(R) \geqslant \frac{n(n-1)}{\| \text { Scal } \|_{1}}
$$

Indeed, Alias proved Inequality (7) with the assumption that $\operatorname{Ric}(M) \geqslant(n+2)(n-1)$. With such an assumption, Inequalities (7) and (6) are exactly the same, but Inequality (6) is valid without any assumption on the Ricci curvature of $M$.

Proof: We use Proposition 3.1 and the fact that the functions $s_{\delta}$ and $c_{\delta}$ are respectively increasing and decreasing.

$$
\begin{aligned}
c_{\delta}(R)^{k} V(M) & \leqslant c_{\delta}(R)^{k-1} \int_{M} c_{\delta}(r) d v_{g} \\
& \leqslant c_{\delta}(R)^{k-1} \int_{M} H s_{\delta}(r) d v_{g} \\
& \leqslant c_{\delta}(R)^{k-2} s_{\delta}(R) \int_{M} H c_{\delta}(r) d v_{g} \\
& \leqslant c_{\delta}(R)^{k-2} s_{\delta}(R) \int_{M} H_{2} s_{\delta}(r) d v_{g} \\
& \leqslant \cdots \\
& \leqslant s_{\delta}(R)^{k} \int_{M} H_{k} d v_{g} .
\end{aligned}
$$

So we have

$$
t_{\delta}(R)^{k} \geqslant \frac{V(M)}{\left\|H_{k}\right\|_{1}}
$$

and equality occurs if and only if $r=R$ for all $x \in M$. Finally, the Hölder inequality gives the result with the $L^{p}$-norm, for all $p \geqslant 1$. 


\section{AN $L^{2}$-APPROACH TO PINCHING}

Let $\left(M^{n}, g\right) \in \mathcal{M}(n, \delta, R, k)$. A first step in the proof of the pinching restults is to prove that the pinching condition

$$
t_{\delta}(R)^{k}<\frac{1}{\left\|H_{k}\right\|_{2 p}}+C
$$

implies that $M$ is closed to a geodesic hypersphere in an $L^{2}$-sense. For this, let's introduce the following functions:

$$
\left\{\begin{array}{l}
\varphi(x)=s_{\delta}^{2}(R)-s_{\delta}^{2}(r) \\
\psi(x)=c_{\delta}(r)\left|Z^{T}\right|
\end{array}\right.
$$

In what follows, we assume that the pinching constant satisfies $C<1$. We prove the following lemma

Lemma 4.1. The pinching condition $\left(P_{C}\right)$ with $C<1$ implies

$$
\|\varphi\|_{2}^{2} \leqslant A_{1} C
$$

where $A_{1}$ is a positive explicit constant depending only on the dimension $n, \delta$ and $\left\|H_{k}\right\|_{2 p}$. Moreover in certain cases, the dependence on $\left\|H_{k}\right\|_{2 p}$ can be replaced by a dependence on $\|H\|_{\infty}$, precisely when

$$
\begin{aligned}
& \text { (1) } \delta>0 \\
& \text { (2) } \delta=0 \text { and } k \geqslant 4 \\
& \text { (3) } \delta=0 \text { and } p \geqslant \frac{n}{2 k} \text {. }
\end{aligned}
$$

Proof: Since $s_{\delta}$ and $c_{\delta}$ are respectively increasing and decreasing, we have

$$
\begin{aligned}
\|\varphi\|_{2}^{2} & \leqslant s_{\delta}^{2}(R) \int_{M}\left(s_{\delta}^{2}(R)-s_{\delta}^{2}(r)\right) \\
& \leqslant s_{\delta}^{2}(R)\left[t_{\delta}^{2}(R)\left(\int_{M} c_{\delta}(r)\right)^{2}-\int_{M} s_{\delta}^{2}(r)\right]
\end{aligned}
$$

By the Hölder inequality, we get

$$
\|\varphi\|_{2}^{2} \leqslant s_{\delta}^{2}(R)\left[t_{\delta}^{2}(R)\left(\int_{M} c_{\delta}(r)\right)^{2}-\frac{1}{\left\|H_{k}\right\|_{2 p}^{2}}\left(\int_{M} H_{k} s_{\delta}(r)\right)^{2}\right]
$$

Now, using Proposition 3.1, we have

$$
\begin{aligned}
\|\varphi\|_{2}^{2} & \leqslant s_{\delta}^{2}(R)\left[t_{\delta}^{2}(R)\left(\int_{M} c_{\delta}(r)\right)^{2}-\frac{1}{\left\|H_{k}\right\|_{2 p}^{2}}\left(\int_{M} H_{k-1} c_{\delta}(r)\right)^{2}\right] \\
& \leqslant s_{\delta}^{2}(R)\left[t_{\delta}^{2}(R)\left(\int_{M} c_{\delta}(r)\right)^{2}-\frac{1}{\left\|H_{k}\right\|_{2 p}^{2} t_{\delta}^{2}(R)}\left(\int_{M} H_{k-1} s_{\delta}(r)\right)^{2}\right]
\end{aligned}
$$


Proposition 3.1 applied $(k-2)$ more times, yields

$$
\begin{aligned}
\|\varphi\|_{2}^{2} & \leqslant s_{\delta}^{2}(R)\left[t_{\delta}^{2}(R)\left(\int_{M} c_{\delta}(r)\right)^{2}-\frac{1}{\left\|H_{k}\right\|_{2 p}^{2} t_{\delta}^{2 k-2}(R)}\left(\int_{M} c_{\delta}(r)\right)^{2}\right] \\
& \leqslant \frac{s_{\delta}^{2}(R)}{t_{\delta}^{2 k-2}(R)}\left(\int_{M} c_{\delta}(r)\right)^{2}\left[t_{\delta}^{2 k}(R)-\frac{1}{\left\|H_{k}\right\|_{2 p}^{2}}\right]
\end{aligned}
$$

Since we assume $\left(P_{C}\right)$ is true with $C<1$, if $\delta>0$, we have

$$
\|\varphi\|_{2}^{2} \leqslant \frac{C}{\delta t_{\delta}^{2 k-2}(R)}\left[1+\frac{2}{\left\|H_{k}\right\|_{2 p}}\right]
$$

Moreover, we have

SO

$$
\frac{1}{t_{\delta}(R)} \leqslant\left\|H_{k}\right\|_{2 p}^{1 / k}
$$

$$
\|\varphi\|_{2}^{2} \leqslant \frac{C}{\delta}\left[\left\|H_{k}\right\|_{2 p}^{\frac{2 k-2}{k}}+2\left\|H_{k}\right\|_{2 p}^{\frac{k-2}{k}}\right]
$$

Since $H_{k}>0$, we have $H \geqslant H_{k}^{1 / k}$ and then $\left\|H_{k}\right\|_{2 p} \leqslant\|H\|_{\infty}^{k}$. Finally, for $k \geqslant 2$, we have

$$
\|\varphi\|_{2}^{2} \leqslant \frac{C}{\delta}\left[\|H\|_{\infty}^{2 k-2}+2\|H\|_{\infty}^{k-2}\right]:=A_{1} C
$$

If $\delta=0$, then

$$
\begin{aligned}
\|\varphi\|_{2}^{2} & \leqslant \frac{C}{R^{2 k-4}}\left[1+\frac{2}{\left\|H_{k}\right\|_{2 p}}\right] \\
& \leqslant C\left[\left\|H_{k}\right\|_{2 p}^{\frac{2 k-4}{k}}+2\left\|H_{k}\right\|_{2 p}^{\frac{k-4}{k}}\right]:=A_{1} C,
\end{aligned}
$$

If, in addition, $4 \leqslant k \leqslant n$, by the same argument as above, $A_{1}$ depends on $n$ and $\|H\|_{\infty}$.

Moreover, for $\delta=0$, we have the following lower bound for the $k$-th mean curvature (see [6] page 221)

$$
\int_{M} H_{k}^{n / k} d v_{g} \geqslant \omega_{n}
$$

where $\omega_{n}$ is the volume of the $n$-dimensional Euclidean sphere. Then, for $p \geqslant \frac{n}{2 k}$, we have

$$
\omega_{n}^{k / n} \leqslant\left\|H_{k}\right\|_{2 p} \leqslant\|H\|_{\infty}^{k} .
$$

So the dependence on $\left\|H_{k}\right\|_{2 p}$ can be replaced by a dependence on $\|H\|_{\infty}$.

Lemma 4.2. The pinching condition $\left(P_{C}\right)$ implies

$$
\|\psi\|_{2}^{2} \leqslant A_{2} C
$$

where $A_{2}$ is a positive explicit constant depending only on $n$ and $\|H\|_{\infty}$. 
Proof: Since $c_{\delta}(r) \leqslant 1$, we have

$$
\begin{aligned}
\|\psi\|_{2}^{2} & \leqslant \int_{M}\left|Z^{T}\right|^{2} \leqslant \int_{M}|Z|^{2}-\langle Z, \nu\rangle^{2} \\
& \leqslant \int_{M} s_{\delta}^{2}(r)-\frac{1}{\left\|H_{k}\right\|_{2 p}^{2}}\left(\int_{M} H_{k}\langle Z, \nu\rangle\right)^{2}
\end{aligned}
$$

By Lemma 2.2, we have

$$
\begin{aligned}
\|\psi\|_{2}^{2} & \leqslant t_{\delta}^{2}(R)\left(\int_{M} c_{\delta}(r)\right)^{2}-\frac{1}{\left\|H_{k}\right\|_{2 p}^{2}}\left(\int_{M} c_{\delta}(r) H_{k-1}\right)^{2} \\
& \leqslant t_{\delta}^{2}(R)\left(\int_{M} c_{\delta}(r)\right)^{2}-\frac{1}{t_{\delta}^{2}(R)\left\|H_{k}\right\|_{2 p}^{2}}\left(\int_{M} s_{\delta}(r) H_{k-1}\right)^{2}
\end{aligned}
$$

Using Proposition 3.1, we get

$$
\begin{aligned}
\|\psi\|_{2}^{2} & \leqslant t_{\delta}^{2}(R)\left(\int_{M} c_{\delta}(r)\right)^{2}-\frac{1}{t_{\delta}^{2}(R)\left\|H_{k}\right\|_{2 p}^{2}}\left(\int_{M} c_{\delta}(r) H_{k-2}\right)^{2} \\
& \leqslant t_{\delta}^{2}(R)\left(\int_{M} c_{\delta}(r)\right)^{2}-\frac{1}{t_{\delta}^{4}(R)\left\|H_{k}\right\|_{2 p}^{2}}\left(\int_{M} c_{\delta}(r) H_{k-2}\right)^{2} \\
& \leqslant \cdots \\
& \leqslant t_{\delta}^{2}(R)\left(\int_{M} c_{\delta}(r)\right)^{2}-\frac{1}{t_{\delta}^{2 k-2}(R)\left\|H_{k}\right\|_{2 p}^{2}}\left(\int_{m} c_{\delta}(r)\right)^{2} \\
& \leqslant \frac{1}{t_{\delta}^{2 k-2}(R)}\left(t_{\delta}^{2}(R)-\frac{1}{\left\|H_{k}\right\|_{2 p}^{2}}\right)
\end{aligned}
$$

So the pinching condition $\left(P_{C}\right)$ with $C<1$ implies

$$
\begin{aligned}
\|\psi\|_{2}^{2} & \leqslant \frac{C}{t_{\delta}^{2 k-2}(R)}\left(1+\frac{2}{\left\|H_{k}\right\|_{2 p}}\right) \\
& \leqslant C\left\|H_{k}\right\|_{2 p}^{\frac{2 k-2}{k}}\left(1+\frac{2}{\left\|H_{k}\right\|_{2 p}}\right) \\
& \leqslant C\left(\left\|H_{k}\right\|_{2 p}^{\frac{2 k-2}{k}}+2\left\|H_{k}\right\|_{2 p}^{\frac{k-2}{k}}\right) \\
& \leqslant C\left(\|H\|_{\infty}^{2 k-2}+2\|H\|_{\infty}^{k-2}\right):=A_{2} C,
\end{aligned}
$$

The next step to prove Theorems 1 and 2 is to get $L^{\infty}$-estimates from these $L^{2}$-estimates. For this, we will use Lemma 2.3.

\section{Proof of Therorem 1}

The proof of Theorem 1 is an immediate consequence of the following three lemmas 
Lemma 5.1. For any $\varepsilon>0$, there exists $K_{\varepsilon}$ depending on $n$ and $\|H\|_{\infty}$ so that if $\left(P_{K_{\varepsilon}}\right)$ is true, then

$$
\phi(M) \subset B\left(p_{0}, R\right) \backslash B\left(p_{0}, R-\varepsilon\right) .
$$

Moreover, $K_{\varepsilon} \longrightarrow 0$ when $\|H\|_{\infty} \longrightarrow+\infty$ or $\varepsilon \longrightarrow 0$.

Proof: We showed in [21] that the function $\varphi$ satisfies

$$
\varphi^{2 k-2} \Delta \varphi^{2} \leqslant \operatorname{div}(\omega)+\left(\alpha_{1}+k \alpha_{2}\right) \varphi^{2 k-1}+\left(\beta_{1}+k \beta_{2}\right) \varphi^{2 k-2},
$$

where $\omega$ is a 1 -form, $\alpha_{1}, \alpha_{2}, \beta_{1}$ and $\beta_{2}$ some nonnegative constants depending on $n, \delta$ and $\|H\|_{\infty}$. We can apply Lemma 2.3 to the function $\varphi$ with $l=1$ and $m=2$. We deduce that if $\|\varphi\|_{\infty}>\varepsilon$ then there exists a constant $L$ such that

$$
\|\varphi\|_{\infty} \leqslant L\|\varphi\|_{2}
$$

On the other hand, by Lemma 4.1, we know that if the pinching condition $\left(P_{C}\right)$ is satisfied for $C \leqslant 1$, then

$$
\|\varphi\|_{2}^{2} \leqslant A_{1} C \text {. }
$$

Take $C=K_{\varepsilon}=\inf \left\{1, \frac{\varepsilon^{2}}{L^{2} A_{1}}\right\}$. This choice implies

$$
\|\varphi\|_{\infty} \leqslant \varepsilon
$$

that is, $t_{\delta}^{2}(R)-t_{\delta}^{2}(r) \leqslant \varepsilon$. Finally, we can choose $K_{\varepsilon}$ smaller in order to have $R-r \leqslant \varepsilon$.

The second lemma is due to B. Colbois and J.F. Grosjean (see [7]).

Lemma 5.2. Let $x_{0}$ be a point of the sphere $S(0, R)$ of $\mathbb{R}^{n+1}$. Assume that $x_{0}=R u$ where $u \in \mathbb{S}^{n}$. Now let $\left(M^{n}, g\right)$ be a compact, connected and oriented $n$-dimensional Riemannian manifold without boundary isometrically immersed by $\phi$ into $\mathbb{R}^{n+1}$ so that

$$
\phi(M) \subset\left(B\left(p_{0}, R+\eta\right) \backslash B\left(p_{0}, R-\eta\right)\right) \backslash B\left(x_{0}, \rho\right)
$$

with $\rho=4(2 n-1) \eta$ and suppose there exists a point $p \in M$ so that $\langle Z, u\rangle(p) \geqslant 0$. Then there exists $y_{0} \in M$ so that the mean curvature satisfies $\left|H\left(y_{0}\right)\right|>\frac{1}{4 n \eta}$.

Remark. Note that in [7], it is supposed that $\langle Z, u\rangle(p)>0$, but the condition $\langle Z, u\rangle(p) \geqslant 0$ is sufficient.

In [21], we give a corresponding lemma for the spherical case.

Lemma 5.3. Let $x_{0}$ be a point of the sphere $S\left(p_{0}, R\right)$ of an open hemisphere of $\mathbb{S}^{n+1}(\delta)$. Let $\left(M^{n}, g\right)$ be a compact, connected and oriented $n$ dimensional Riemannian manifold without boundary isometrically immersed by $\phi$ into this open hemisphere of $\mathbb{S}^{n+1}(\delta)$ so that

$$
\phi(M) \subset\left(B\left(p_{0}, R\right) \backslash B\left(p_{0}, R-\eta\right)\right) \backslash B\left(x_{0}, \rho\right)
$$


with $\rho$ such that

$$
t_{\delta}(R / 2)-t_{\delta}((R-\rho) / 2)=4(2 n-1) \eta
$$

Then there exists two constants $D$ and $E$ depending on $n, \delta$ and $R$ such that if $\eta \leqslant D$, then there exists $y_{0} \in M$ so that

$$
\left|H\left(y_{0}\right)\right| \geqslant \frac{E}{8 n \eta} .
$$

\section{Proof of Theorem 1:}

The proof for $\delta=0$ is an immediate consequence of Lemmas 5.1 and 5.2. Indeed, let $\varepsilon>0$. By Lemma 5.1, there exists $K_{\varepsilon}$ such that if $\left(P_{K_{\varepsilon}}\right)$ is true, then

$$
\phi(M) \subset B\left(p_{0}, R\right) \backslash B\left(p_{0}, R-\varepsilon\right) .
$$

Let $x=R u \in S(0, R)$ and assume that $\phi(M) \cap B(x, \varepsilon)=\emptyset$. Since $R$ is the extrinsic radius, there exists a point $p \in M$ such that $\langle Z, u\rangle(p) \geqslant 0$. If $\left(P_{C_{\varepsilon}}\right)$ is true with $C_{\varepsilon}=K_{\frac{\varepsilon}{4(2 n-1)}}$ and $\varepsilon<\frac{2}{3\|H\|_{\infty}}$, then by Lemma 5.2, there exists $y_{0} \in M$ so that

$$
\left|H\left(y_{0}\right)\right| \geqslant \frac{1}{4 n\left(\frac{\varepsilon}{4(2 n-1)}\right)} \geqslant \frac{2 n-1}{n \varepsilon}>\|H\|_{\infty},
$$

which is a contradiction. Finally, $\phi(M) \cap B(x, \varepsilon) \neq \emptyset$ which completes the proof for $\delta=0$.

For $\delta \neq 0$, let $\varepsilon>0$. We set $0<\eta:=\inf \left\{D, \varepsilon, \frac{\gamma(\varepsilon)}{8(2 n-1)}\right\}$, where

$$
\gamma(\varepsilon)=t_{\delta}\left(\frac{R}{2}\right)-t_{\delta}\left(\frac{R-\varepsilon}{2}\right) \text {. }
$$

Note that $\gamma$ is an increasing smooth function with $\gamma(0)=0$. From Lemma 5.1, there exists $C_{\varepsilon}=K_{\eta}$ such that $\left(P_{C_{\varepsilon}}\right)$ implies

$$
R-r \leqslant \eta \leqslant \varepsilon
$$

That's the first point of Theorem 1. Assume that $\varepsilon<\gamma^{-1}\left(\frac{2 E}{3\|H\|_{\infty}}\right)$. Suppose there exists $x \in S\left(p_{0}, R\right)$ such that $B(x, \varepsilon) \cap M=\emptyset$. Since $\gamma(\varepsilon) \geqslant 4(2 n-1) \eta$, by Lemma 5.3, there exists a point $y_{0} \in M$ so that

$$
\left|H\left(y_{0}\right)\right| \geqslant \frac{E}{8 n \eta} \geqslant \frac{(2 n-1) E}{n \gamma(\varepsilon)}>\|H\|_{\infty} .
$$

Hence a contradiction and $B(x, \varepsilon) \cap M \neq \emptyset$.

Moreover, for $\delta=0$ or $\delta>0$, by Lemma 5.1, $C_{\varepsilon} \longrightarrow 0$ when $\|H\|_{\infty} \longrightarrow 0$ or $\varepsilon \longrightarrow 0$. 


\section{Proof of Theorem 2}

We first need the following lemma

Lemma 6.1. For any $\varepsilon>0$, there exists $K_{\varepsilon}$ depending on $n$ and $\|B\|_{\infty}$ so that if $\left(P_{K_{\varepsilon}}\right)$ is true, then

$$
\|\psi\|_{\infty} \leqslant \varepsilon
$$

Moreover, $K_{\varepsilon} \longrightarrow 0$ when $\|B\|_{\infty} \longrightarrow+\infty$ or $\varepsilon \longrightarrow 0$.

Proof: We proved in 21] that the function $\psi$ satisfies

$$
\psi^{2 k-2} \Delta \psi^{2} \leqslant \operatorname{div}(\omega)+\left(\alpha_{3}+k \alpha_{4}\right) \psi^{2 k-1}+\left(\beta_{3}+k \beta_{4}\right) \psi^{2 k-2} .
$$

Now applying Lemma 2.3 with $l=1$ and $m=2$, we get that for any $\eta>0$, there exists $L$ depending on $n$ and $\|B\|_{\infty}$ so that if $\|\psi\|_{\infty}>\eta$ then

$$
\|\psi\|_{\infty} \leqslant L\|\psi\|_{2}
$$

From Lemma 4.1, we know that if $\left(P_{C}\right)$ holds, then

$$
\|\psi\|_{2}^{2} \leqslant A_{2} C \text {. }
$$

Let $\varepsilon>0$, we set $C=K_{\varepsilon}=\inf \left\{1, \frac{\varepsilon^{2}}{L^{2} A_{2}}\right\}$. For this choice of $C$ we get

$$
\|\psi\|_{\infty} \leqslant \varepsilon \text {. }
$$

This completes the proof.

\section{Proof of Theorem2:}

We consider the following map

$$
\begin{aligned}
& F: M \longrightarrow S\left(p_{0}, R\right) \\
& x \longmapsto \exp _{p_{0}}\left(R\left(\mathrm{~d} \exp _{p_{0}}\right)^{-1}(\bar{\nabla} r)\right) .
\end{aligned}
$$

We proved in 21] that for any uniatry vector $u \in T_{x} M$

$$
\left.|| \mathrm{d}_{x} F(u)\right|^{2}-1\left|\leqslant \frac{1}{s_{\delta}^{2}(r)}\right| s_{\delta}^{2}(R)-s_{\delta}^{2}(r) \mid+\frac{s_{\delta}^{2}(R)}{c_{\delta}(r) s_{\delta}^{3}(r)}\|\psi\|_{\infty} .
$$

From Lemma 5.2, we know that for any $\eta>0$, there exists a constant $K_{\eta}$ so that $\left(P_{K_{\eta}}\right)$ implies $\|\psi\|_{\infty} \leqslant \eta$. Moreover, by Theorem 1, there exist $C_{\eta}$ depending on $n, \delta,\|H\|_{\infty}$ and $\eta$ so that $\left(P_{C_{\eta}}\right)$ implies $R-r \leqslant \eta$. We set $C_{\eta}^{\prime}=\inf \left\{C_{\eta}, K_{\eta}\right\}$. Then, since $R$ is bounded by a constant depending only on $n, \delta,\left\|H_{k}\right\|_{2 p}$ and $\|H\|_{\infty}$ there exist three positive constants $A_{3}, A_{4}$ and $A_{5}$ depending on $n, \delta,\left\|H_{k}\right\|_{2 p}$ and $\|H\|_{\infty}$ so that

$$
\begin{aligned}
\left.|| \mathrm{d}_{x} F(u)\right|^{2}-1 \mid & \leqslant A_{3}\|R-r\|_{\infty}+A_{4}\|\psi\|_{\infty} \\
& \leqslant A_{3} \eta+A_{4} \eta \leqslant A_{5} \eta
\end{aligned}
$$

Now, choosing $\eta=\frac{\theta}{A_{5}}$, we get

$$
\left.|| \mathrm{d}_{x} F(u)\right|^{2}-1 \mid \leqslant \theta .
$$


For $\theta \in] 0,1\left[\right.$, by (10), $F$ is a local diffeomorphism from $M$ to $S\left(p_{0}, R\right)$. Since for $n \geqslant 2, S\left(p_{0}, R\right)$ is simply connected, $F$ is a diffeomorphism. Moreover, the relation (10) says that $F$ is a quasi-isometry.

Remark. If $\delta=0$ and $p \geqslant \frac{n}{2 k}$, we saw that

$$
\omega_{n}^{k / n} \leqslant\left\|H_{k}\right\|_{2 p} \leqslant\|H\|_{\infty}^{k} \leqslant \frac{1}{n^{k / 2}}\|B\|_{\infty}^{k},
$$

so there is no dependence on $\left\|H_{k}\right\|_{2 p}$.

\section{REFERENCES}

1. L.J. Alias, An integral formula for compact hypersurfaces of space forms and its applications, J. Aust. Math. Soc. 74 (2003), 239-248.

2. J. Aminov, The exterior diameter of an immersed Riemannian manifold, Math. USSR-Sb 21 (1973), 449-454, AMS translation.

3. E. Aubry, Variétés de courbure de ricci presque minorée: inégalités géométriques optimales et stabilité des variétés extrémales, Ph.D. thesis, Université Joseph Fourier, Grenoble, 2003.

4. C. Baikoussis and T. Koufogiorgos, The diameter of an immersed Riemannian manifold with bounded mean curvature, J. Austral. Soc. (Series A) 31 (1981), 189-192.

5. J.L. Barbosa and A.G. Colares, Stability of hypersurfaces with constant $r$-th mean curvature, Ann. Global Anal. Geom. 15 (1997), 277-297.

6. Y.D. Burago and V.A. Zalgaller, Geometric inequalities, Springer-Verlag, Berlin, 1988.

7. B. Colbois and J.F. Grosjean, A pinching theorem for the first eigenvalue of the Laplacian on hypersurfaces of the Euclidean space, to appear in Comment. Math. Helv.

8. T.H. Colding, Large manifolds with positive Ricci curvature, Invent. Math. 124 (1996), 193-214.

9. _ Shape of manifolds with positive Ricci curvature, Invent. Math. 124 (1996), 175-191.

10. C.B. Croke, An eigenvalue pinching theorem, Invent. Math. 68 (1982), 253-256.

11. S. Deshmukh, An integral formula for compact hypersurfaces in a Euclidean space and its applications, Glasgow Math. J. 34 (1992), 309-311.

12. J.F. Grosjean, Upper bounds for the first eigenvalue of the Laplacian on compact submanifolds, Pac. J. Math. 206 (2002), no. 1, 93-111.

13. _ Extrinsic upper bounds for the first eigenvalue of elliptic operators, Hokkaido Math. J 33 (2004), no. 2, 219-239.

14. T. Hasanis and D. Koutroufiotis, Immersions of bounded mean curvature, Arc. Math. 33 (1979), 170-171.

15. E. Heintze, Extrinsic upper bounds for $\lambda_{1}$, Math. Ann. 280 (1988), 389-402.

16. C.C. Hsiung, Some integral formulae for closed hypersurfaces, Math. Scand. 2 (1954), 286-294.

17. S. Ilias, Un nouveau résultat de pincement de la première valeur propre du Laplacien et conjecture du diamètre pincé, Ann. Inst. Fourier 43 (1993), no. 3, $843-863$.

18. S. Montiel and A. Ros, Compact hypersurfaces: the alexandrov theorem for higher order mean curvature, Pitman Monographs Surveys Pure Appl. Math. 52 (1991), 279-286, in honor of M.P. Do Carmo; edited by B. Lawson and K. Tenenblat. 
19. P. Petersen, On eigenvalue pinching in positive Ricci curvature, Invent. Math. 138 (1999), 1-21.

20. R.C. Reilly, On the first eigenvalue of the Laplacian for compact submanifolds of Euclidean space, Comment. Math. Helv. 52 (1977), 525-533.

21. J. Roth, Extrinsic radius pinching for hypersurfaces of space forms, To appear in Diff. Geom. Appl.

22. T. Vlachos, A characterisation for geodesic spheres in space forms, Geom. Dedic. 68 (1997), 73-78.

23. J.Y. Wu, A diameter pinching sphere theorem for positive Ricci curvature, Proc. Amer. Math. Soc. 107 (1989), no. 3, 797-802. 